\title{
La modélisation du transport solide par charriage à l'aide d'un modèle aux éléments finis
}

\author{
J. M. Tanguy $(1,2,3)$ G. Dhatt $(2,3)$ M. Frenette $\left({ }^{3}\right)$, P. Monadier ( $\left.{ }^{1}\right)$ \\ ( $\left.{ }^{1}\right)$ Service technique central des Ports maritimes et des Voies navigables (Compiègne) \\ $\left.{ }^{2}\right)$ Université de technologie de Compiègne \\ (3) Université Laval (Québec)
}

Sous l'action des courants, les particules solides qui tapissent le fond des fleuves, des estuaires et des mers peuvent être mises en mouvement et être entraînées sur des distances plus ou moins grandes. Ces remaniements sédimentaires, qui se traduisent par des érosions ou des dépôts sont susceptibles d'affecter l'équilibre d'un site et conditionnent son aménagement.

Depuis quelques années, on assiste à un engouement croissant pour l'emploi de modèles mathématiques, capables de simuler les évolutions des fonds sous l'effet des courants. Ils autorisent en effet l'étude de plusieurs variantes d'un projet en un temps très court et permettent d'analyser leurs répercussions sur le milieu.

C'est dans cette optique que nous nous sommes intéressés au transport de matériaux et plus particulièrement au transport par charriage, qui constitue un mode de transport essentiel dans de nombreux sites. Rappelons que celui-ci consiste en le roulement, le glissement ou la saltation de particules sur le fond: c'est donc un phénomène essentiellement bidimensionnel horizontal. Pour simuler un tel processus, nous avons conçu un modèle numérique bidimensionnel de charriage en utilisant la Méthode des Eléments Finis. Cette méthode a été choisie car elle offre une grande souplesse d'utilisation : elle permet en effet la prise en compte de géométries très complexes, par l'intermédiaire d'éléments de dimensions et d'orientations différentes. Ceci la rend donc tout à fait adaptée à des sciences comme l'hydraulique et la sédimentologie.

Dans la suite de cet article, nous présentons la formulation mathématique des modèles utilisés, la formulation numérique correspondante par éléments finis, ainsi que l'étude de l'évolution des fonds aux alentours d'un épi résultant de mesures effectuées en laboratoire.

\section{Modelisation of solid bed-load transport with the finite element method}

The Finite Element method gained remarkable popularity during the last decade in Fluid Mechanics, because of its great flexibility, required to modelise complex domains found in nature.

The authors present a two dimensional Finite Element model of bed load transport, which allows simulation of spatial-temporal variations of bed profile. The formulation is fully discussed, including remedies introduced to treat unsymetrical diffusion leading to unrealistic bed profile evolutions. A flume experiment, related to bed profile evolution near a spur-dike has been undertaken in order to validate the present model. 


\section{Les modèles mathématiques}

\subsection{Le modèle hydrodynamique}

Les particules solides étant yéhiculées par l'écoulement, il convient de disposer en tout premier lieu d'un modèle hydrodynamique capable de déterminer le champ de vitesse en tout point du domaine. Celui-ci est du type " en eaux peu profondes " issu des équations de SaintVenant. Il s'agit donc d'un modèle intégré sur la verticale, régi par le système d'équations :

Mouvement :

$$
\begin{aligned}
\frac{\partial u_{i}}{\partial t}+ & u_{j} \frac{\partial u_{i}}{\partial x_{j}}+g \frac{\partial h}{\partial x_{i}}=-g \frac{n^{2}|V| u_{i}}{(H+h)^{4 / 3}}+ \\
& +F_{c i}+\frac{\partial \sigma_{i j}}{\partial x_{j}}+\frac{C_{w} \rho_{a}}{\rho(H+h)}|W| W_{i} \quad \begin{array}{l}
i=1,2 \\
j=1,2
\end{array}
\end{aligned}
$$

Continuité :

$$
\frac{\partial h}{\partial t}+\frac{\partial(H+h) u_{j}}{\partial x_{j}}=0 \quad j=1,2
$$

où : $x_{i}=\langle x, y\rangle=$ abscisses vers l'Est et ordonnées vers le Nord, $u_{i}=\langle u, v\rangle=$ vitesses moyennes intégrées sur la verticale, $g=$ accélération de la gravité, $n=$ coefficient de Manning, $h=$ niveau de la surface libre, $H=$ niveau du fond, $F_{c i}=\left(f_{u},-f_{v}\right)=$ force de Coriolis, $\sigma_{t}=$ contraintes visqueuses, $C_{W}=$ coefficient de trâ̂née du vent, $W=$ vitesse du vent (à $10 \mathrm{~m}$ ), $\rho_{a}=$ masse spécifique de l'air.

\subsection{Le modèle de charriage}

Le transport par charriage est approché par l'équation d'état suivante :

Transport :

$$
q=q\left(u_{i}, H, h, \lambda, \rho_{s}, d\right)
$$

où $\lambda$ est la porosité du matériau, $\rho_{s}$ sa masse volumique et $d$ le diamètre moyen des particules constituant le fond.

L'évolution du fond $H(x, y)$ est définie par l'équation de conservation de la matière sédimentaire :

Conservation :

$$
(1-\lambda) \frac{\partial H}{\partial t}+\frac{\partial q_{j}}{\partial x_{j}}=0 . \quad j=1,2 .
$$

La majorité des formules de transport (éq. (3)) sont des formules à seuil qui peuvent s'exprimer sous la forme :

$$
\begin{array}{lll}
q=\alpha\left(\tau-\tau_{c}\right)^{\beta} & & \tau_{c} \leqslant \tau<\tau_{c}^{\prime} \\
q=0 & \tau<\tau_{c} \\
q=q_{\max } & \tau \geqslant \tau_{c}^{\prime}
\end{array}
$$

où $\tau_{c}$ est la contrainte critique de décollement des matériaux du fond, $\tau_{c}^{\prime}$ le début du phénomène de suspension et $\tau$ la contrainte hydrodynamique exercée par l'écoulement sur le fond, donnée par :

$$
\vec{\tau}=\rho g \frac{n^{2}|V| \vec{V}}{(H+h)^{4 / 3}} .
$$

Une loi de type Shields a été utilisée pour le calcul de $\tau_{c}$ :

$$
\tau_{c}=A\left(\rho_{s}-\rho\right) g d .
$$

Précisons que la contrainte moyenne $\tau$, calculée par le modèle hydrodynamique est corrigée pour prendre en compte l'effet des formes de fond (rides, dunes). Ceci se fait par l'intermédiaire du coefficient de Strickler global $k(k=1 / n)$, fonction de la rugosité des grains $\left(k_{r}\right)$ et de la rugosité de forme $\left(k_{s f}\right)$. La relation utilisée est celle proposée par Hengelund et Hansen [4].

$$
\begin{aligned}
k_{s f}= & 11,3 \sqrt{g}\left[\frac{d}{(H+h)^{4 / 3}}\right]^{1 / 2} \times \\
& \times\left(0,06+0,16\left[\frac{(H+h) J}{d}\right]^{2}\right)^{5 / 8} J^{-5 / 8} \\
k_{r}= & \frac{26,4}{d^{1 / 6}}\left[\frac{d}{H+h}\right]^{1 / 24}
\end{aligned}
$$

où $J$ est la pente de la ligne d'énergie et $k$ est donné par : $k=k_{s f}$ pour $k_{s f}>k_{r}$, et $k=k_{r}$ dans le cas contraire.

\section{La formulation par éléments finis}

\subsection{Le modèle hydrodynamique}

Le champ de vitesse est obtenu en utilisant un élément triangulaire à 6 nœuds avec une approximation quadratique pour le calcul des vitesses et une approximation linéaire pour les niveaux d'eau. Le schéma implicite d'Euler est couplé à la méthode de Newton-Raphson pour obtenir une solution temporelle [2], [3].

\subsection{Le modèle de charriage}

La variation du niveau du fond est obtenue en utilisant un triangle à 3 nœuds pour la résolution de l'équation de conservation de la matière, couplée à une relation algébrique de transport $q$ (éq. (5)).

La forme variationnelle associée à l'équation (4) s'écrit :

$$
\begin{aligned}
W= & \sum W_{e}=\sum \int_{A^{e}} H^{*}\left(\dot{H}+\frac{\partial q_{j}}{\partial x_{j}}\right) \mathrm{d} A=0 \\
& \forall t, H^{*}(x, y) \text { et } \dot{H}=(1-\lambda) \frac{\partial H}{\partial t} \quad j=1,2
\end{aligned}
$$

où $H^{*}$ est une fonction de pondération de type Galerkine. La forme variationnelle élémentaire a pour expression :

$$
\begin{aligned}
& W_{e}=\left\langle\delta H_{n}\right\rangle \times \\
& \times\left[\frac{1}{24} \text { dét }(J)\left[\begin{array}{lll}
2 & 1 & 1 \\
1 & 2 & 1 \\
1 & 1 & 2
\end{array}\right]\left\{\dot{H}_{n}\right\}+\frac{1}{6}(A+B)\left\{\begin{array}{l}
1 \\
1 \\
1
\end{array}\right]\right]
\end{aligned}
$$

avec

$$
\begin{array}{ll} 
& A=\left(y_{2}-y_{3}\right) q_{x 1}+\left(y_{3}-y_{1}\right) q_{x 2}-\left(y_{1}-y_{2}\right) q_{x 3} \\
& B=\left(x_{3}-x_{2}\right) q_{y 1}+\left(x_{1}-x_{3}\right) q_{y 2}-\left(x_{2}-x_{1}\right) q_{y 3} \\
\text { et dét }(J) \text { est le double de l'aire du triangle réel. }
\end{array}
$$




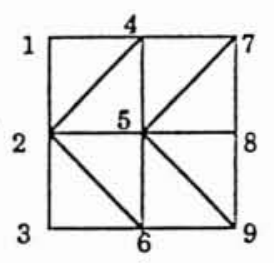

$a$

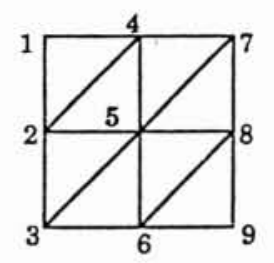

b

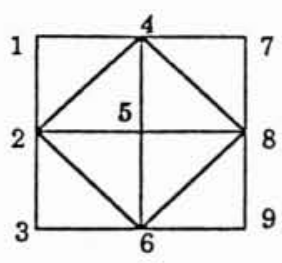

$c$

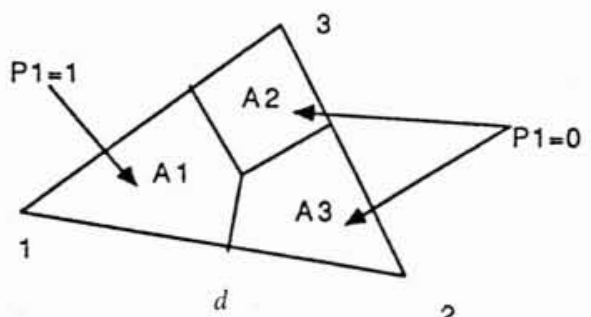

1. $a$ : Type $1 ; b:$ Type $2 ; c$ : Type 3.

Les tests effectués sur cette formulation standard nous ont conduit à apporter 2 types de modification, afin d'éliminer les oscillations numériques parasites de la solution.

Matrice masse diagonale: Considérons le schéma discrétisé résultant de l'équation (9). En formulation monodimensionnelle, le schéma s'écrit pour un maillage uniforme de longueur " $L$ ", si « $i$ " désigne la position spatiale :

$$
\left(\dot{H}_{i+1}+4 \dot{H}_{i}+\dot{H}_{i-1}\right) / 6+\left(q_{i+1}-q_{i-1}\right) / 2 L=0 .
$$

Cette expression peut être mise sous la forme :

$\dot{H}_{i}+\left(\dot{H}_{i+1}-2 \dot{H}_{i}+\dot{H}_{i-1}\right) / 6+\left(q_{i+1}-q_{i-1}\right) / 2 L=0$

qui revient à introduire une diffusion supplémentaire sur le terme temporel et en fait à résoudre l'équation suivante :

$$
\frac{\partial}{\partial t}\left(H+\frac{L^{2}}{6} \frac{\partial^{2} H}{\partial x^{2}}\right)_{i}+\left(\frac{\partial q}{\partial x}\right)_{i}=0 .
$$

Pour conserver un schéma non diffusif, nous utiliserons une matrice masse diagonale, ce qui conduit au schéma :

$$
(\dot{H})_{i}+\left(\frac{\partial q}{\partial x}\right)_{i}=0
$$

Cette approche a été étendue à la formulation bidimensionnelle.

Fonctions poids modifiées: En deux dimensions, il est apparu que l'utilisation de fonctions poids classiques de type Galerkine conduisait à affecter à chaque nœud d'un élément, un poids identique, amenant des évolutions de fonds dissymétriques. L'exemple suivant illustre ce phénomène.

Considérons un domaine soumis à un état de sollicitation caractérisé par les valeurs nodales suivantes:

$$
\begin{aligned}
& q_{1}=q_{2}=q_{3}=Q_{1} ; \\
& q_{4}=q_{5}=q_{6}=Q_{2} ; \\
& q_{7}=q_{8}=q_{9}=Q_{3} .
\end{aligned}
$$

Pour les 3 types de maillage représentés par les schémas $1 a, 1 b, 1 c$, les évolutions de fond $(\Delta H / \Delta \mathrm{t})$, qui résultent de l'application de l'équation (11) ont pour valeurs aux nœuds 4,5 et 6 :
Type 1

$$
\begin{aligned}
& \text { Nœud } 4 \text { et } 6:\left(-2 Q_{1}+Q_{2}+Q_{3}\right) / 3 \\
& \text { Nœud 5: } \quad\left(-Q_{1}-Q_{2}+2 Q_{3}\right) / 3
\end{aligned}
$$

Type 2

$$
\begin{aligned}
& \text { Nœud } 4 \text { et } 6:\left(-2 Q_{1}+Q_{2}+Q_{3}\right) / 3 \\
& \text { Nœud } 5: \quad\left(-Q_{1}+Q_{3}\right) / 3 .
\end{aligned}
$$

Type 3

$$
\begin{aligned}
& \text { Nœud } 4 \text { et } 6:\left(-Q_{1}+Q_{3}\right) / 3 \\
& \text { Nœud } 5: \quad\left(-Q_{1}+Q_{3}\right) / 3 .
\end{aligned}
$$

Il apparaît donc une dissymétrie transversale dont l'importance dépend du maillage utilisé. Nous avons recherché une répartition nodale qui prendrait en compte la distorsion des éléments, ainsi que l'influence de chaque nœud d'une manière plus acceptable. Pour maintenir la symétrie, nous avons choisi les fonctions $H$, $H^{*}$ et $q$ suivantes :

$$
\begin{aligned}
& H=\left\langle P_{i}\right\rangle\left\{H_{i}\right\} ; H^{*}=\left\langle P_{i}\right\rangle\left\{H_{i}^{*}\right\} ; \\
& q=\langle 1-\xi-\eta \xi \eta\rangle\left\{q_{i}\right\} ; i=1,3 .
\end{aligned}
$$

$P_{i}$ sont des constantes sur les 3 sous-domaines de l'élément. Par exemple, $P_{1}=1$ sur $A_{1}$ et $P_{1}=0$ ailleurs (cf. fig. 1d).

Finalement, la forme variationnelle élémentaire a pour expression :

$$
\begin{aligned}
& W_{e}=\left\langle\delta H_{n}\right\rangle \times \\
& \times\left[\operatorname{dét~}(J)\left[\begin{array}{ccc}
P_{1} & 0 & 0 \\
0 & P_{2} & 0 \\
0 & 0 & P_{3}
\end{array}\right]\left\{\dot{H}_{n}\right\}+(A+B)\left\{\begin{array}{l}
P_{1} \\
P_{2} \\
P_{3}
\end{array}\right]\right]
\end{aligned}
$$

où :

$P_{1}=\left(x_{p}+y_{p}\right) / 2 ; P_{2}=\left(1-x_{p}\right) / 2 ; P_{3}=\left(1-y_{p}\right) / 2$

avec :

$$
\begin{aligned}
x_{p}= & 1 / 2-(1 / 2)\left(x_{2} x_{3}+y_{2} y_{3}\right)\left[x_{3}\left(x_{3}-x_{2}\right)+\right. \\
& \left.+y_{3}\left(y_{3}-y_{2}\right)\right] /\left(x_{2} y_{3}-x_{3} y_{2}\right)^{2} \\
y_{p}= & (1 / 2)\left(x_{2}^{2}+y_{2}^{2}\right)\left[x_{3}\left(x_{3}-x_{2}\right)+\right. \\
& \left.+y_{3}\left(y_{3}-y_{2}\right)\right] /\left(x_{2} y_{3}-x_{3} y_{2}\right)^{2}
\end{aligned}
$$

où $P(x, y)$ est le centre du cercle inscrit du triangle réel et $x_{i}, y_{i}(i=1,3)$ les coordonnées des sommets de ce triangle. 

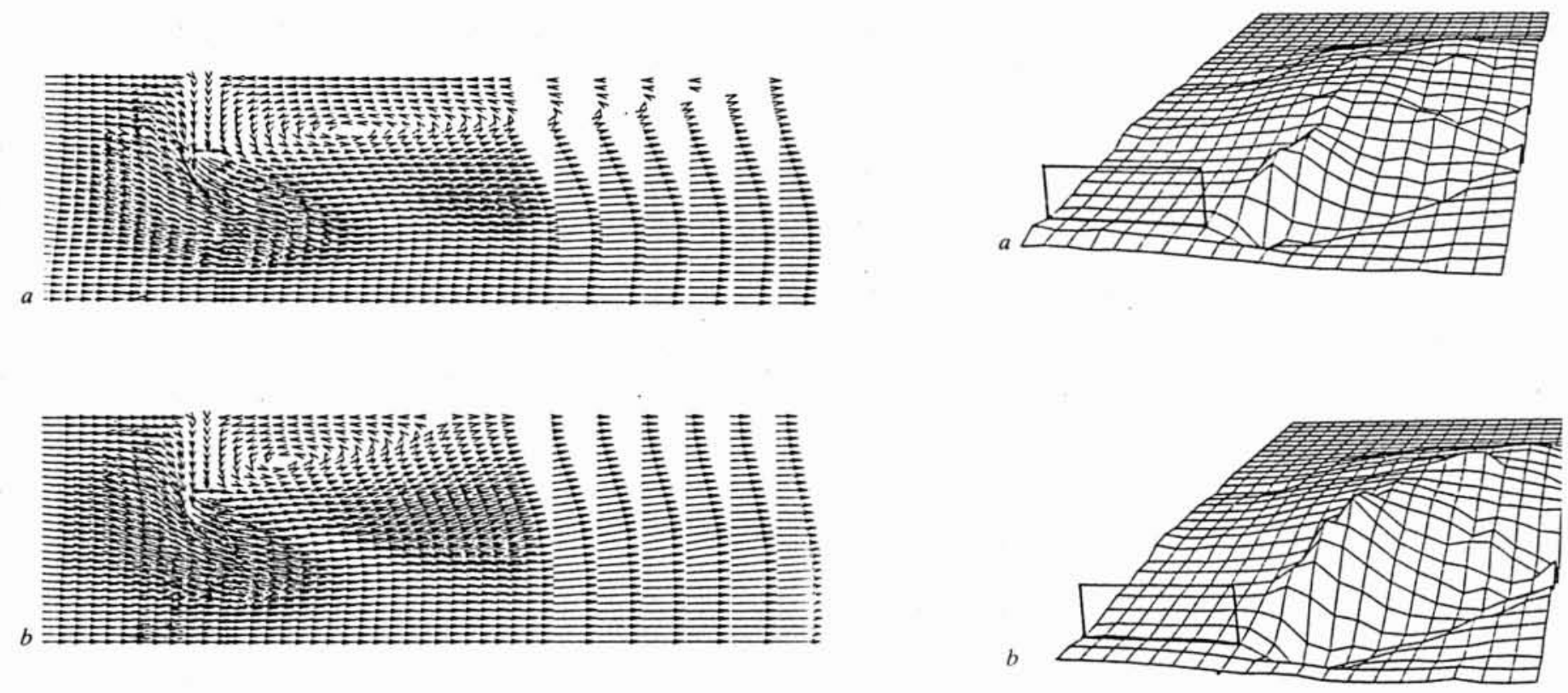

2. $a$ : Champ de vitesses initial. $b$ : Champ de vitesses final.

3. Essai en canal. Etat du fond: $a: T=12 \mathrm{~h} ; b: T=$ $48 \mathrm{~h}$.
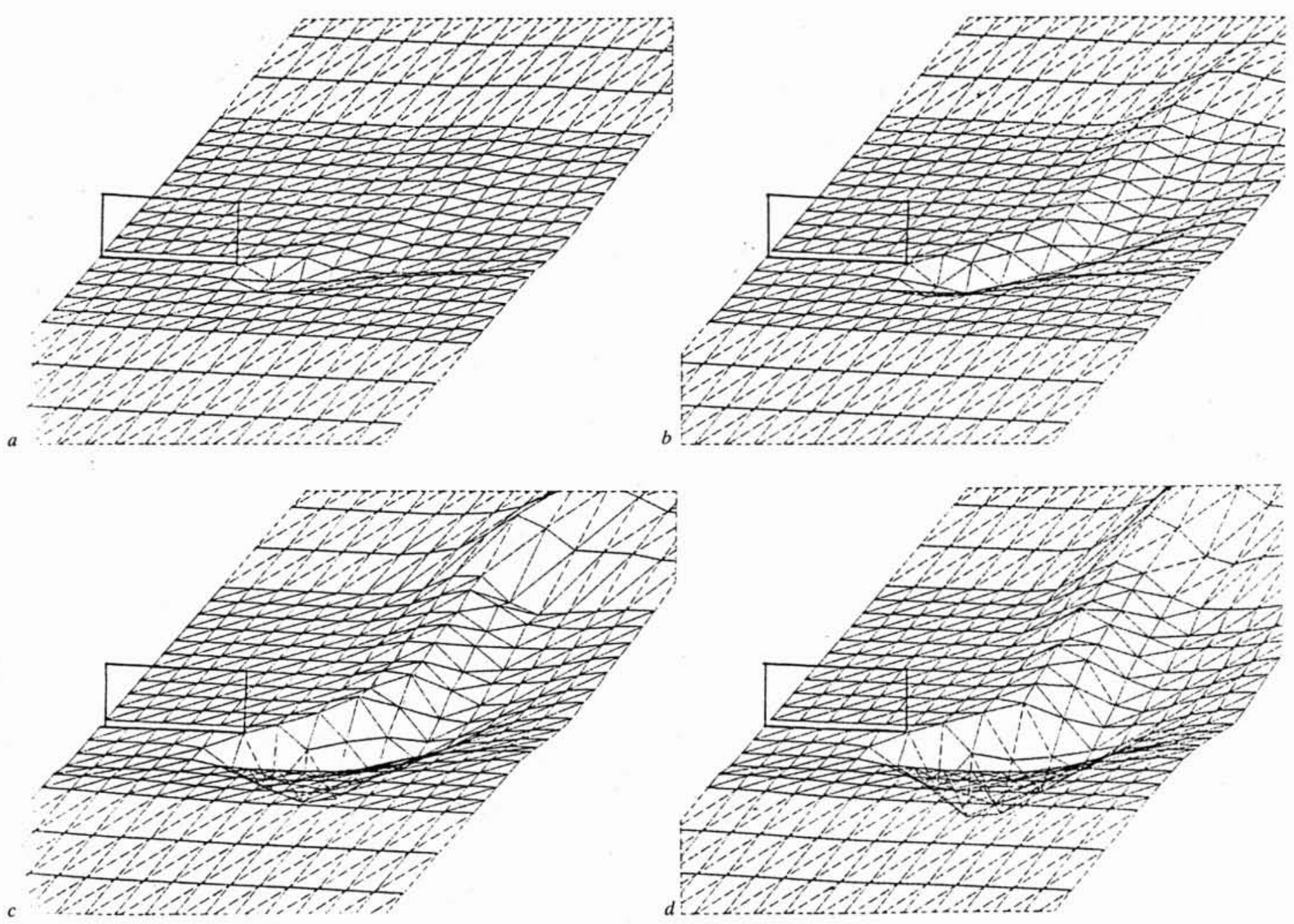

4. Simulation numérique. Etat du fond: $a: T=6 \mathrm{~h} ; b$ : $T=12 \mathrm{~h} ; c: T=24 \mathrm{~h} ; d: T=48 \mathrm{~h}$. 


\section{Etude de cas}

Pour étudier l'interaction de l'écoulement sur un domaine à fonds mobiles, nous avons réalisé en laboratoire un canal long de $20 \mathrm{~m}$ (dont $10 \mathrm{~m}$ utiles) et large de $1,85 \mathrm{~m}$, au fond duquel est disposé un lit de sable sur une profondeur de $0,20 \mathrm{~m}$. Le sable a un diamètre moyen de $0,32 \mathrm{~mm}$, une masse volumique de $2665 \mathrm{~kg} / \mathrm{m}^{3}$ et une porosité de 0,4. Perpendiculairement aux parois de ce canal, donc à l'écoulement, nous avons disposé un épi d'une longueur de $0,60 \mathrm{~m}$. Les conditions d'écoulement ont été choisies de manière à ce que le transport n'ait lieu que par charriage. Ainsi, tout au long de l'essai, le débit liquide a été maintenu à $60 \mathrm{l} / \mathrm{s}$, et la hauteur d'eau à l'aval de l'installation à une valeur de $0,20 \mathrm{~m}$. Aucun apport de matériaux n'a été fait.

Au niveau hydrodynamique, le déroulement de l'essai a mis en évidence une forte interaction entre le fond et l'écoulement. Les mesures de vitesses effectuées indiquent qu'elles diminuent sensiblement au cours du temps. Les schémas de la figure 2, qui représentent les champs de vitesse reproduits par le modèle numérique, au démarrage de l'essai et après stabilisation des fonds, montrent très nettement l'influence de l'évolution des fonds sur l'hydrodynamique du système. Il apparaît que l'écoulement se régularise progressivement sous l'effet de l'adaptation de la morphologie des fonds.

Pour ce qui est de l'évolution temporelle du domaine, la figure 3 donne un aperçu de l'état des fonds mesurés sur le modèle physique après $12 \mathrm{~h}$ et $48 \mathrm{~h}$ d'essai. On peut ainsi apercevoir, au voisinage de l'épi, une fosse d'érosion qui se développe au cours du temps, jusqu'à atteindre un profil d'équilibre. Les matériaux provenant de cette fosse sont déplacés par le courant vers l'aval où ils se déposent dans les zones à plus basses vitesses, ce qui explique les dépôts observés dans le canal. Les schémas de la figure 4 illustrent les résultats provenant de la simulation numérique, obtenus par la relation de Meyer-Peter. On constate la bonne concordance entre le modèle physique et le modèle numérique. Les zones de dépôt et d'érosion sont convenablement localisées. En outre, la profondeur maximale d'érosion calculée avoisine $11 \mathrm{~cm}$ contre $12 \mathrm{~cm}$ mesurés dans le canal, et la hauteur de dépôt atteint $8 \mathrm{~cm}$ contre $7 \mathrm{~cm}$ mesurés.

Il convient de noter que la zone d'érosion maximale se situe bien dans les zones à forts gradients de transport, qui ne correspondent pas avec celles de grandes vitesses. Enfin, le modèle numérique indique une absence totale de mouvement des particules à l'aval de l'épi, dans la zone où se développe le tourbillon : ce qui est en accord avec les observations faites dans le canal.

\section{Conclusion}

Nous avons présenté un nouveau modèle bidimensionnel de transport de matériaux par charriage. Celui-ci consiste en le couplage original d'un modèle hydrodynamique, qui comprend une actualisation spatio-temporelle du coefficient de rugosité, d'une équation d'état et d'un modèle d'évolution du fond. Grâce à l'utilisation de techniques numériques originales, adaptées à sa formulation par éléments finis, ce modèle donne toutes satisfactions et a été testé avec succès sur un essai en laboratoire. $\mathrm{Ce}$ dernier consistait en l'étude de l'évolution des fonds aux alentours d'un épi. Les résultats obtenus par le modèle numérique sont très comparables à la réalité.

\section{Remerciements}

Nous tenons à remercier TAO Simulations Inc. (Québec) pour la mise à disposition du module hydrodynamique Meflu.

\section{Bibliographie}

[1] Dhatt G., Touzot G., Une présentation de la méthode des éléments finis, Maloine Editeur, Paris, 1981.

[2] Cochet J. F., Dhatt G., Touzot G., « Comparison of Explicit and Implicit Methods Applied to Finite Element Models of Tidal Problems". 3rd Int. Conf. on Finite Elements in Flow Problems. Banff, Canada, June 1980.

[3] LeClerc M. et al., « Modélisation des écoulements de l'Archipel de Montréal par éléments finis ". Revue internationale des Sciences de l'eau, 1987, vol. 3, n², p. 41-56.

[4] Engelund F., Hansen A., A Monograph on Sediment Transport in Alluvial Streams. Technical University of Denmark, 1967.

[5] Yalin M. S., Mechanics of Sediment Transport, 2nd Ed., Pergamon Press, 1977. 